\title{
Direction of Arrival Estimation with a Novel Single-Port Smart Antenna
}

\author{
Chen Sun \\ School of Electrical and Electronic Engineering, Nanyang Technological University, Nanyang Avenue, Singapore 639798 \\ Email: csun@ieee.org
}

Nemai C. Karmakar

Department of Electrical and Computer Systems Engineering, Monash University, Clayton, VIC 3800, Australia

Email:nemai.karmakar@eng.monash.edu.au

Received 19 May 2003; Revised 13 November 2003

\begin{abstract}
A novel direction of arrival (DOA) estimation technique that uses the conventional multiple-signal classification (MUSIC) algorithm with periodic signals is applied to a single-port smart antenna. Results show that the proposed method gives a highresolution (1 degree) DOA estimation in an uncorrelated signal environment. The novelty lies in that the MUSIC algorithm is applied to a simplified antenna configuration. Only 1 analogue-to-digital converter (ADC) is used in this antenna, which features low power consumption, low cost, and ease of fabrication. Modifications to the conventional MUSIC algorithm do not bring much additional complexity. The proposed technique is also free from the negative influence by the mutual coupling among antenna elements. Therefore, it offers an economical way to extensively implement smart antennas into the existing wireless mobile communications systems, especially at the power consumption limited mobile terminals such as laptops in wireless networks.
\end{abstract}

Keywords and phrases: smart antennas, direction finding, multiple-signal classification, digital beamforming array antennas, parasitic array antenna.

\section{INTRODUCTION}

The wireless industry is growing 6 times faster than the fixed line services. The number of the mobile subscribers has grown from 0.5 billion in 1999 to 1.3 billion in 2003. The global revenue from the wireless market has been doubled from 1999 to 2003 as more than 400 billion dollars [1]. Wireless communication systems are evolving from the second generation systems to the third and fourth generation systems, which will provide high data rate multimedia services as video transmission. New value-added services such as the position location (PL) services for emergency calls, the fraud detection, intelligent transportation systems, and so forth, are also coming into reality $[2,3,4]$.

Efforts have been focused on the developing coding, protocol, and modulation of the second generation system, while the role of the antennas has been overlooked. Recently, in the form of adaptive array or smart antennas, they attract interests among researchers. Deployed at the base station of the existing wireless infrastructures, they bring an outstanding capacity improvement to the frequency resource limited radio communication systems by an efficient frequency reuse scheme, for example, space division multiple-access (SDMA) scheme [5]. The direction-finding ability of the smart anten- nas is important to the PL services. Furthermore, they also benefit the design of the routing protocol in recently thriving ad hoc networks [6].

Various beamforming and direction of arrival (DOA) estimation algorithms have been designed [1]. Simulations and experiments carried out by many researchers have shown the abilities of these algorithms. Most of these algorithms are designed based on the digital beamforming (DBF) array antennas (Figure 1). Signals received by the individual antenna elements are converted down into baseband signals, digitized and fed into a digital signal processing (DSP) chip where the algorithms reside. However, RF circuit branches connected to the array elements, analog to digital converters (ADCs) and the baseband DSP chip consume a considerable amount of DC power. Furthermore, each channel connected to the array elements has the same structure, so the cost of fabrication increases with the number of array elements [7]. All these factors make the DBF antenna arrays unsuitable for low power consumption and low cost systems and hinder the mass implementation of the smart antenna technologies. It could be too costly to equip a DBF array antenna at batterypowered laptops or mobile-computing terminals within a wireless network. 


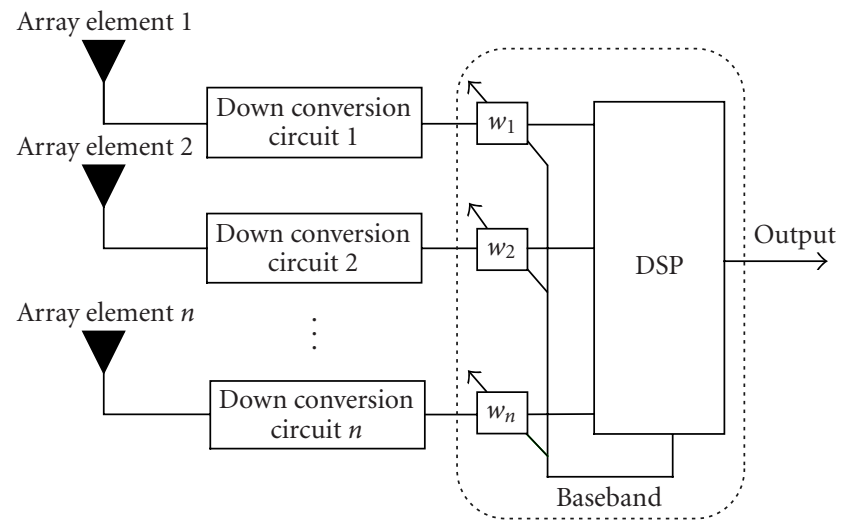

FIgURE 1: Functional block diagram of the DBF array antennas.

To circumvent the problems with the DBF array antennas, we propose a novel smart antenna structure, which has only $1 \mathrm{RF}$ port. It is a parasitic array antenna [8]. One central element connected to the sole RF port and a number of surrounding switched and reactively loaded parasitic elements form the array (Figure 2). We call the antenna "switched and reactively loaded parasitic array (SARLPA)." Since the system has only 1 RF port connected to the active central element and 1 subsequent down conversion circuit, it obviously consumes much less power than a DBF antenna array. Beam steering of parasitic array antennas is achieved by either selecting different sets of parasitic elements with a switching control circuitry in a manner similar to switched beam antennas (called "switched parasitic antennas" $[9,10,11,12])$, or by controlling the reactive loading at the parasitic elements to steer the beam continuously (called "reactively controlled directive arrays" [13] or electronically steerable parasitic array radiator (ESPAR) $[14,15,16,17,18])$. The SARLPA antenna is half wavelength in diameter. It may not be suitable for mobile phones, but could be easily mounted on mobile terminals in wireless networks. For example, the physical dimension of the array operating at $2.4 \mathrm{GHz}$ is $62.25 \mathrm{~mm}$, which is suitable for mounting on laptops.

In this paper, a high-resolution direction finding using the multiple-signal classification (MUSIC) algorithm with periodic signals is employed on this single-port smart antenna. Activating different beam patterns by switching on and off different parasitic elements, while the signal is periodically transmitted, the received scalar signal from the RF port can be stacked into a vector over one period. The beam pattern is shifted with a predefined angle. So, for the application of MUSIC, it is sufficient to know these angular shifts, without knowing exactly the response to one subset. This is similar to a uniform circular or linear antenna array in which we know the phase shift between consecutive antenna responses, without having to know those responses exactly. The MUSIC algorithm is carried out with the constructed signal vector over 1 period and the modified steering vector based on the beam pattern angle shift. Simulation justifies the proposal. Our heuristic study demonstrates the feasibil-

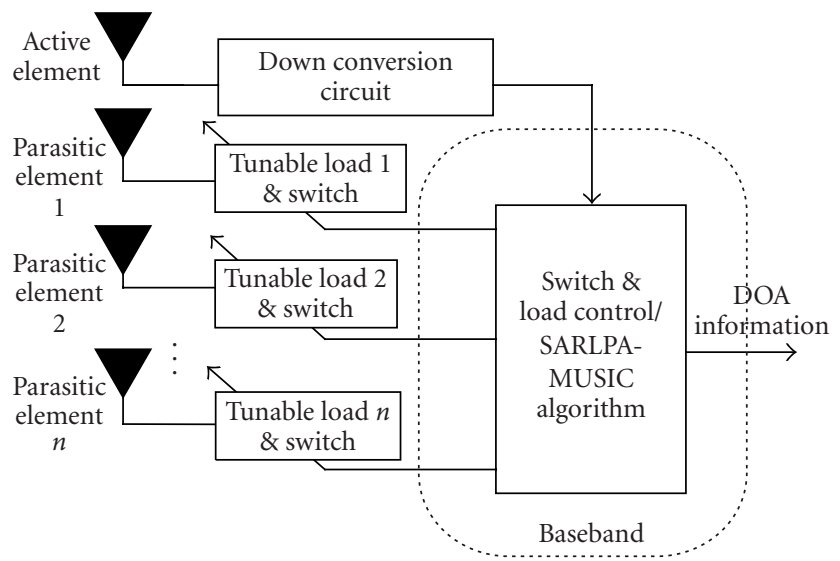

FIGURE 2: Functional block diagram of the SARLPA.

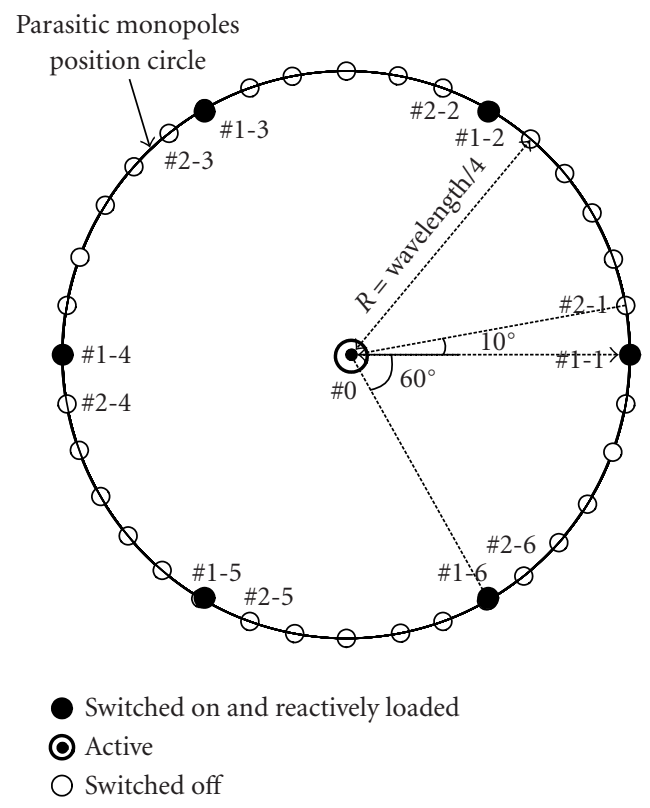

Figure 3: Top view of a 37-element SARLPA.

ity of employing the eigenstructure-based direction-finding algorithm with a parasitic array that has only $1 \mathrm{RF}$ port.

The paper is organized as follows: Section 2 presents the configuration and the working principle of the SARLPA. The algorithm for DOA estimation is presented in Section 3. Results are produced in Section 4, followed by conclusions and recommendations in Section 5.

\section{DESIGN OF THE ANTENNA}

Figure 2 shows the functional block diagram of a SARLPA antenna. Figure 3 gives the top view of the antenna. One active central element (monopole) is surrounded by 36 parasitic elements on a circle of radius $\mathrm{R}$ on the circular grounded baseplate. The length of each monopole $(\mathrm{L})$ and the radius (R) are one quarter wavelength $(\lambda / 4)$ of the transmitting RF signal. The baseplate transforms the monopoles with their 


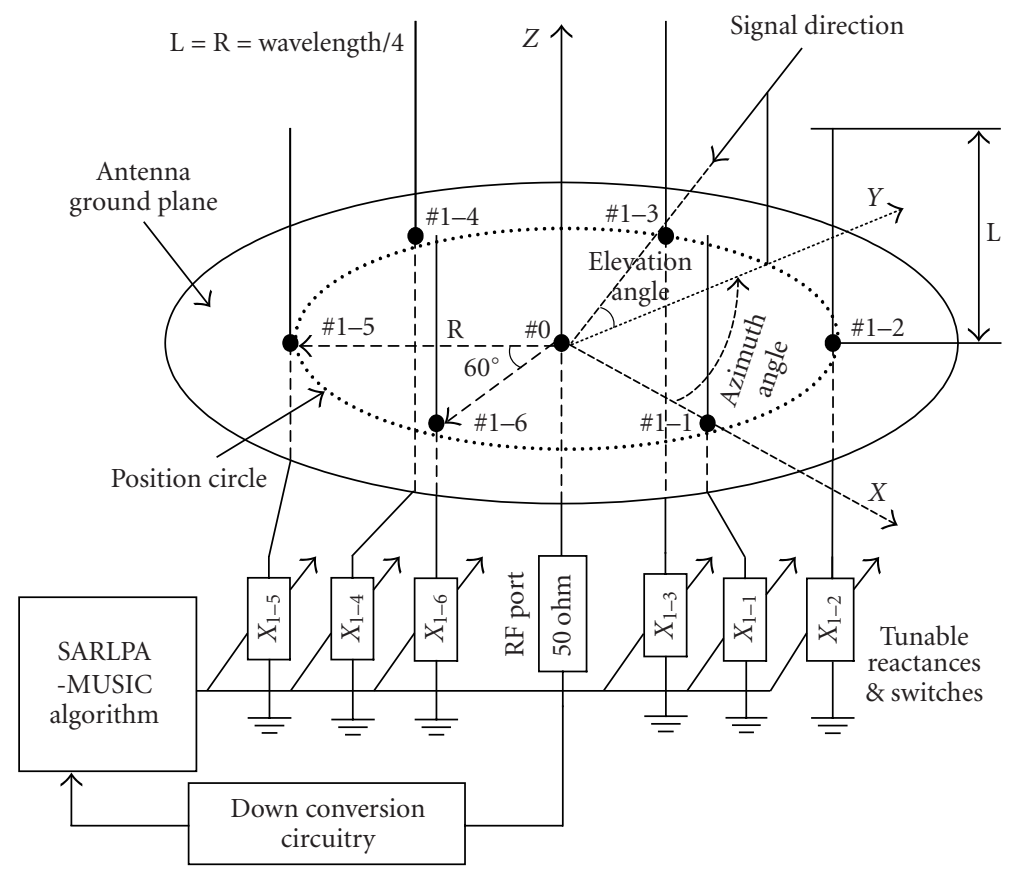

FIGURE 4: Equivalent representation of an SARLPA when subset \#1 is activated. The DOA in the azimuth plane of the impinging wave is also shown in the figure.

images to dipoles with a length of $2 \mathrm{~L}$. The central monopole is connected to an RF receiver and each parasitic monopole is loaded with a tunable reactor and is controlled by a switch. The circuitry could be simply implanted as in [19]. Since both the switches and the tunable reactances are implemented at each individual parasitic elements, the antenna provides great flexibility of operation. It can be reconfigured into a switched parasitic array or a reactively controlled directive array antenna.

As shown in Figure 3, the 36 parasitic monopoles are grouped into 6 subsets. Each subset consists of 6 parasitic elements equispaced on the circle of radius $\mathrm{R}$. The central monopole is designated as \#0, whereas the parasitic element $\# m-n(m=1, \ldots, 6 ; n=1, \ldots, 6)$ refers to the $n$th element of the subset \#m. The antenna is operated in such a way that at any time only 1 subset is switched on and the rest of the subsets are switched off. The switched-off elements do not contribute to beam forming and can be viewed as "transparent" to other switched-on elements. This is realized either by switching the parasitic monopoles to open circuit as in $[9,10]$, or by tuning the load reactance value to make the element invisible electrically [20]. The central element is always activated as signals pass through the sole RF port. Therefore, at any time, only 6 parasitic monopoles from the selected subset and 1 active central monopole are in operation. Figure 4 gives an equivalent representation of each subset comprised of the active central monopole and the 6 parasitic monopoles.

During operation, the first subset (parasitic monopoles $\# 1-1, \# 1-2$, \#1-3, \#1-4, \#1-5, and \#1-6) is selected. After a certain period, the subset \#2 (parasitic monopoles \#2-1, \#2-
2, \#2-3, \#2-4, \#2-5, and \#2-6) is selected. As this process continues, the antenna array is virtually rotating anticlockwise on its vertical axis. The loads at parasitic monopoles are tuned in the same manner for all subsets. We have selected 36 parasitic and 1 active monopoles to satisfy the modified MUSIC algorithm as applied to the proposed SARLPA configuration. For practical applications, the number of the monopoles could be chosen with the tradeoff between the complexity and the performance (number of waves to be estimated, beam pattern directivity, etc.).

At any configuration, the antenna has the form of a reactively controlled directive array or an ESPAR. We explain the working principle based on the equivalent structure as the subset \#1 is activated as shown in Figure 4. The beam forming of a SARLPA is different from that for a DBF. The antenna generates a directional beam based on tuning load reactances $\left(x_{1}, x_{2}, \ldots, x_{6}\right)$ on the parasitic monopoles. Signals received or transmitted from the central RF port excite the passive parasitic monopoles with substantial induced mutual currents on them. In the following discussion, we assume that the antenna is working in the transmitting mode. Vectors $\bar{I}$ and $\bar{V}$ represent the currents and the voltages on the monopoles, respectively:

$$
\begin{gathered}
\bar{I}=\left[\begin{array}{lllllll}
i_{0} & i_{1} & i_{2} & i_{3} & i_{4} & i_{5} & i_{6}
\end{array}\right]^{T}, \\
\bar{V}=\left[\begin{array}{lllllll}
v_{0} & v_{1} & v_{2} & v_{3} & v_{4} & v_{5} & v_{6}
\end{array}\right]^{T} .
\end{gathered}
$$

Superscript $T$ represents the transpose. Mutual admittances are represented by the matrix $\mathbf{Y}$ with each entity $y_{i j}$ denotes mutual admittance between two elements [16]. There, the 
induced mutual currents are represented by mutual admittances,

$$
\bar{I}=\mathbf{Y} \bar{V}=\left[\begin{array}{lllllll}
y_{00} & y_{01} & y_{02} & y_{03} & y_{04} & y_{05} & y_{06} \\
y_{10} & y_{11} & y_{12} & y_{13} & y_{14} & y_{15} & y_{16} \\
y_{20} & y_{21} & y_{22} & y_{23} & y_{24} & y_{25} & y_{26} \\
y_{30} & y_{31} & y_{32} & y_{33} & y_{34} & y_{35} & y_{36} \\
y_{40} & y_{41} & y_{42} & y_{43} & y_{44} & y_{45} & y_{46} \\
y_{50} & y_{51} & y_{52} & y_{53} & y_{54} & y_{55} & y_{56} \\
y_{60} & y_{61} & y_{62} & y_{63} & y_{64} & y_{65} & y_{66}
\end{array}\right] \times\left[\begin{array}{c}
v_{0} \\
v_{1} \\
v_{2} \\
v_{3} \\
v_{4} \\
v_{5} \\
v_{6}
\end{array}\right]
$$

The voltages at monopoles are

$$
v_{0}=v_{s}-z_{0} i_{0}
$$

(for the active central element.) $z_{0}=50 \Omega$ is the impedance at the RF port.

$$
v_{l}=-j x_{l} i_{l}
$$

(for parasitic elements $l=1, \ldots, 6$ ), $v_{s}$ represents the transmitted voltage signal source with amplitude and phase from the driving RF port at the central element \#0. We represent (3) and (4) with a matrix form,

$$
\bar{V}=\left[\begin{array}{lllllll}
v_{s} & \mathbf{0} & \mathbf{0} & \mathbf{0} & \mathbf{0} & \mathbf{0} & \mathbf{0}
\end{array}\right]^{T}-\mathbf{X} \bar{I}=v_{s} U_{1}-\mathbf{X} \bar{I},
$$

and define

$$
\begin{aligned}
\mathbf{X} & =\operatorname{diag}\left[z_{0}, j x_{1}, \ldots, j x_{6}\right], \\
U_{1} & =\left[\begin{array}{lllllll}
1 & 0 & 0 & 0 & 0 & 0 & 0
\end{array}\right]^{T} .
\end{aligned}
$$

Combining (2) and (5), we get

$$
\bar{I}=\mathbf{Y} \bar{V}=\mathbf{Y}\left(v_{s} U_{1}-\mathbf{X} \bar{I}\right) .
$$

After a simple mathematical manipulation, we obtain the equation as

$$
\overline{\mathbf{I}}=v_{s}\left(\mathbf{I}_{(7)}+\mathbf{Y X}\right)^{-1} \mathbf{Y} U_{1}=v_{s} E_{1}
$$

In (8), $\left(\mathbf{I}_{(7)}+\mathbf{Y X}\right)^{-1} \mathbf{Y} U_{1}$ is represented by a $(7 \times 1)$ dimensional vector $E_{1} . \mathbf{I}_{(7)}$ is a $(7 \times 7)$-dimensional identity matrix. The far field radiation pattern is formed by the superposition of radiation patterns of all monopoles on the antenna ground plane $[8,21]$. The far field current signal with its amplitude and phase in direction $\phi_{k}$ is

$$
y_{\mathrm{far}}(t)=\alpha\left(\phi_{k}\right) \bar{I}=v_{s} \alpha\left(\phi_{k}\right) E_{1} .
$$

We assume that signals arrive in the azimuth plane. $k$ is the signal sources index. $\alpha\left(\phi_{k}\right)$ is the steering vector corresponding to the signal impinging direction $\phi_{k}$ and is given based on the array geometry (Figure 3 ). Please note that only one parasitic set and the active element are in operation,

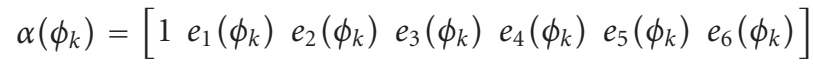

$$
\begin{aligned}
& e_{i}\left(\phi_{k}\right)=\exp \left\{j \frac{2 \pi}{\lambda} R \cos \left(\phi_{k}-\frac{2 \pi}{6}(i-1)\right)\right\} \\
& =\exp \left\{j \frac{\pi}{2} \cos \left(\phi_{k}-\frac{\pi}{3}(i-1)\right)\right\} \quad(i=1, \ldots, 6) .
\end{aligned}
$$

Setting $v_{s}$ in (9) as unity, we can get the array factor $\hat{\alpha}\left(\phi_{k}\right)$ of the SARLPA antenna when any one subset is activated as

$$
\hat{\alpha}\left(\phi_{k}\right)=\alpha\left(\phi_{k}\right) E_{1}
$$

Thus, the far field signal in (9) is represented as

$$
y_{\mathrm{far}}(t)=\hat{\alpha}\left(\phi_{k}\right) v_{s}
$$

According to the reciprocity theory for radiation patterns [22], the received voltage signal $u(t)$ at the RF port is

$$
u(t)=\hat{\alpha}\left(\phi_{k}\right) s_{k}(t)+n(t),
$$

$s_{k}(t)$ represents the $k$ th far field incident current wave with amplitude and phase in the azimuth plane. $n(t)$ is the additive white Gaussian noise (AWGN) with the power of $\sigma^{2}$. When there are totally $K$ signal sources, the output from the $\mathrm{RF}$ port is

$$
u(t)=\sum_{k=1}^{K} \hat{\alpha}\left(\phi_{k}\right) s_{k}(t)+n(t) .
$$

Since $\hat{\alpha}\left(\phi_{k}\right)$ is dependent on the reactance matrix $\mathbf{X}$, the desired antenna beam patterns are formed by adjusting the reactance values. In practice, the variable reactance can be easily achieved by changing the bias voltage of Schottky [22] or varactor diodes loaded at parasitic monopoles.

In our proposed DOA estimation technique, the antenna is not used for adaptive beamforming, though it still has the ability to do so. Desired beam patterns are formed to explore the spatial information of the signal sources.

\section{FORMING OF THE CORRELATION MATRIX WITH SINGLE-PORT SARLPA}

The MUSIC algorithm, proposed by Schmidt [23], is a relatively simple and high-resolution eigenstructure approach. It has been widely used as a model-based approach for DOA estimation and source location problems. There are also many improved forms, such as the root MUSIC algorithm and the beamspace MUSIC algorithm [24]. All these algorithms require snapshots of signals from antenna linear or circular array elements to form the signal correlation matrix.

It is obvious that we cannot apply the conventional MUSIC algorithm to the SARLPA antenna, because it has only $1 \mathrm{RF}$ port. Forming the signal sample vector is impossible at one time. This is the distinction between the MUSIC algorithm based on a single-port smart antenna and the 
conventional MUSIC algorithm based on a DBF array antenna. However, we can see from the explanation in the following part that the received signals from the RF port contain signal sources direction information, and the signal correlation matrix can still be formed.

In operation, 6 subsets of parasitic elements are selected sequentially as indicated in Figure 3. The beam pattern rotates $10^{\circ}$ for 6 times consecutively. Therefore, the antenna is virtually rotated. The sample signal vector $U(t)=$ $\left[u_{1}(t), \ldots, u_{m}(t), \ldots, u_{6}(t)\right]^{T}$ is constructed with signal samples from the RF output. Each entity $u_{m}(t), m=1, \ldots, 6$ is obtained in the sampling period when the subset $\# m$ is in operation. The maximum number of sampling periods $M$ equals the number of subsets. For the SARLPA antenna with 6 subsets, after rotating $6+1$ times, the beam pattern returns to the original status. The load reactance values of parasitic monopoles are the same for all the sampling periods. $E_{1}$, defined in (8), remains constant as the antenna rotates virtually. However, the signal directions with respect to the antenna $X-Y$ coordinate (Figure 4 ) change as the antenna is virtually rotated. According to (14), the received signal sample at each sampling period is represented as:

$$
\begin{gathered}
u_{m}(t)=\sum_{k=1}^{K} \hat{\alpha}\left(\phi_{k}-\frac{2 \pi}{36}(m-1)\right) s_{k}(t)+n_{m}(t), \\
m=1,2, \ldots, 6
\end{gathered}
$$

For the simplicity, we define

$$
\hat{\alpha}_{m}\left(\phi_{k}\right)=\hat{\alpha}\left(\phi_{k}-\frac{2 \pi}{36}(m-1)\right)
$$

$\hat{\alpha}_{m}\left(\phi_{k}\right)$ contains the $k$ th signal source direction information $\phi_{k} . n_{m}(t)$ is the noise component at the $m$ th sampling period. Suppose each individual signal source transmits periodic signals and the length of 1 signal period is the same as the sampling period. Then received signal samples are the same for all sampling periods. Therefore, the received signal vector $U(t)$ after 6 sampling periods can be written into the following matrix multiplication form:

$$
\begin{aligned}
U(t)=\left[\begin{array}{c}
u_{1}(t) \\
u_{2}(t) \\
u_{3}(t) \\
u_{4}(t) \\
u_{5}(t) \\
u_{6}(t)
\end{array}\right]= & {\left[\begin{array}{cccc}
\hat{\alpha}_{1}\left(\phi_{1}\right) & \hat{\alpha}_{1}\left(\phi_{2}\right) & \cdots & \hat{\alpha}_{1}\left(\phi_{K}\right) \\
\hat{\alpha}_{2}\left(\phi_{1}\right) & \hat{\alpha}_{2}\left(\phi_{2}\right) & \cdots & \hat{\alpha}_{2}\left(\phi_{K}\right) \\
\hat{\alpha}_{3}\left(\phi_{1}\right) & \hat{\alpha}_{3}\left(\phi_{2}\right) & \cdots & \hat{\alpha}_{3}\left(\phi_{K}\right) \\
\hat{\alpha}_{4}\left(\phi_{1}\right) & \hat{\alpha}_{4}\left(\phi_{2}\right) & \cdots & \hat{\alpha}_{4}\left(\phi_{K}\right) \\
\hat{\alpha}_{5}\left(\phi_{1}\right) & \hat{\alpha}_{5}\left(\phi_{2}\right) & \cdots & \hat{\alpha}_{5}\left(\phi_{K}\right) \\
\hat{\alpha}_{6}\left(\phi_{1}\right) & \hat{\alpha}_{6}\left(\phi_{2}\right) & \cdots & \hat{\alpha}_{6}\left(\phi_{K}\right)
\end{array}\right] } \\
& \times\left[\begin{array}{c}
s_{1}(t) \\
s_{2}(t) \\
\vdots \\
s_{K}(t)
\end{array}\right]+\left[\begin{array}{c}
n_{1}(t) \\
n_{2}(t) \\
\vdots \\
n_{6}(t)
\end{array}\right]
\end{aligned}
$$

Equation (17) has the same form as the output from an array antenna with 6 elements. In the first matrix on the right-hand side of the equation, we define each column as vector $\vec{\alpha}\left(\phi_{k}\right)$. It corresponds to the steering vector [25] for a 6-element array antenna as reported in array signal processing literature. But each entity is not a phase delay of a source signal induced when the plane passes the array with an impinging angle relative to the antenna. We call $\vec{\alpha}\left(\phi_{k}\right)$ "SARLPA-direction vector" to differentiate it from the "steering vector" for conventional antenna arrays. The signal correlation matrix at the antenna output is $\mathbf{R}_{U U}$ :

$$
\mathbf{R}_{U U}=E\left(U(t) U^{H}(t)\right)=\frac{1}{N s} U U^{H} .
$$

$E(\cdot)$ is the expected value operator. $U$ without a time argument represents the discrete samples with sample block length $N s$. Superscript $H$ denotes conjugate transpose. We assume that all the noise components $n_{m}(t), m=1, \ldots, 6$ at each sampling period $\# m$ are independent identically distributed (i.i.d.) AWGN with the power of $\sigma^{2}$. $\mathbf{R}_{U U}$ is then represented as

$$
\begin{aligned}
\mathbf{R}_{U U}= & {\left[\begin{array}{llll}
\vec{\alpha}\left(\phi_{1}\right) & \vec{\alpha}\left(\phi_{2}\right) & \cdots & \vec{\alpha}\left(\phi_{K}\right)
\end{array}\right] \times \mathbf{R}_{S S} } \\
& \times\left[\begin{array}{lllll}
\vec{\alpha}\left(\phi_{1}\right) & \vec{\alpha}\left(\phi_{2}\right) & \cdots & \left.\vec{\alpha}\left(\phi_{K}\right)\right]^{H}+\sigma^{2} \mathbf{I}_{(6)}
\end{array}\right.
\end{aligned}
$$

$\mathbf{I}_{(6)}$ is a $(6 \times 6)$-dimensional identity matrix. The source signal correlation matrix $\mathbf{R}_{S S}$ is

$$
\begin{aligned}
\mathbf{R}_{S S}=E( & {\left[\begin{array}{lll}
s_{1}(t) s_{2}(t) & \cdots & s_{k}(t)
\end{array}\right] } \\
& \left.\times\left[\begin{array}{llll}
s_{1}(t) s_{2}(t) & \cdots & s_{k}(t)
\end{array}\right]^{H}\right) .
\end{aligned}
$$

Based on (18), the MUSIC algorithm is executed in a similar procedure as the conventional MUSIC algorithm. Figure 5 gives the flowchart. The SARLPA direction vector is used to obtain the MUSIC spectrum. The algorithm requires the signals to be periodic. That means proposed technique cannot be applied when mobile terminals are transmitting message signals, because message signals are quite indeterministic. It could, however, be implemented in a pilot or a command channel or by assigning periodic codes at message channels. Therefore, we call it "MUSIC algorithm with periodic signals." Its procedure is described below.

(i) Construct the signal sample vector given by $U(t)=$ $\left[u_{1}(t), \ldots, u_{m}(t), \ldots, u_{6}(t)\right] . u_{m}(t), m=1, \ldots, 6$, is the signal sample when the antenna is at sampling period $\# m$. With $U(t)$, we form the signal correlation matrix $\mathbf{R}_{U U}$.

(ii) Eigendecompose the signal correlation matrix $\mathbf{R}_{U U}$, and form the noise subspace $\mathbf{E}_{N}$ with eigenvectors corresponding to the small eigenvalues.

(iii) Evaluate the MUSIC spectrum $p_{M U}$ versus the signal direction $\phi$,

$$
p_{M U}=\frac{1}{\left|\mathbf{E}_{N}^{H} \vec{\alpha}(\phi)\right|^{2}},
$$

$\vec{\alpha}(\phi)$ is the SARLPA direction vector corresponding to the azimuthal looking direction $\phi$. 


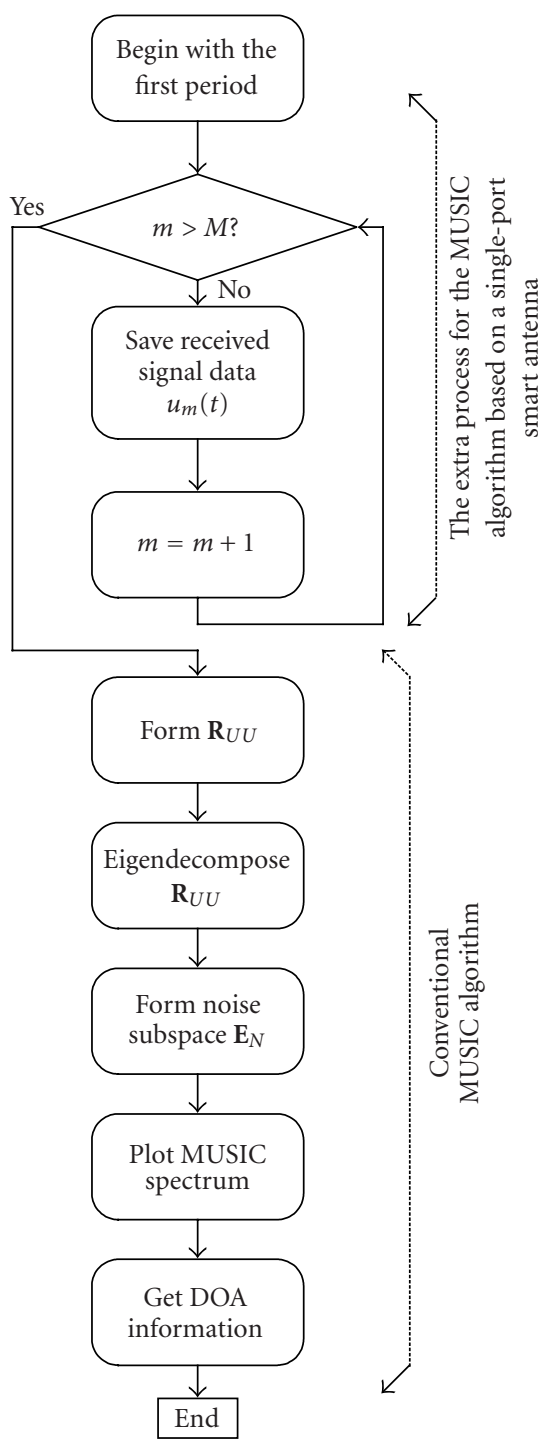

FIGURE 5: Flowchart of the conventional and the proposed MUSIC algorithm and the proposed MUSIC algorithm based on a singleport smart antenna.

(iv) Finally, extract the impinging waves direction information.

Besides the additional procedure for forming the signal sample vector, the proposed technique follows same procedure as that for the conventional MUSIC algorithm. An extra amount of time is required for storing the data to form the signal sample vector.

\section{SIMULATION}

In our simulation, 3 binary phase shift keying (BPSK) signals with equal power levels are randomly generated. The signal impinging directions are set arbitrarily to $120^{\circ}, 150^{\circ}$, and $300^{\circ}$ in the azimuth plane, respectively. During each of the 6 antenna sampling periods, the length of signal samples is 16 bits. This indicates that $N_{s}$ is 16 in (18).

The monopoles on the ground plane of the SARLPA antenna are oriented vertically. The impinging signals are assumed copolarized with the antenna. The cross-polarization coupling typically produced by scatters and reflectors in multipath propagation environment [26] is not considered.

\subsection{Influence of parasitic load reactance}

We study four different reactance load settings to investigate their influence on the performance of the DOA estimation. Table 1 summarizes the reactance values for these 4 cases.

MUSIC spectra with different reactive load settings in uncorrelated signal environments where the signal-to-noiseratio (SNR) is $40 \mathrm{~dB}$ for each individual signal source are presented and explained as follows.

\subsubsection{Case 1. Equal load reactance of $30 \Omega$}

In Case 1, we set all the parasitic load reactances to $30 \Omega$. The antenna exhibits a nearly omnidirectional beam pattern. It could not be virtually rotated in a way as described previously. The MUSIC spectrum is plotted in Figure 6. From the figure, we can observe, that the signal direction could not be extracted.

\subsubsection{Case 2. Ordered but unequal load reactances of $0 \Omega$ and $30 \Omega$}

In Case 2, we set the load to $x_{1}=0 \Omega, x_{2}=30 \Omega, x_{3}=0 \Omega$, $x_{4}=30 \Omega, x_{5}=0 \Omega$, and $x_{6}=30 \Omega$. The beam pattern for rotation is shown in Figure 7. The MUSIC spectrum is plotted in Figure 8. It also cannot give the direction information. From the simulation studies in Cases 1 and 2, we conclude that we should not set the loads "ordered," which will result in insufficient beam patterns for rotation.

\subsubsection{Case 3. Random load reactances of $0 \Omega$ and $30 \Omega$}

In Case 3, we arbitrarily set $x_{1}=0 \Omega, x_{2}=30 \Omega, x_{3}=0 \Omega$, $x_{4}=30 \Omega, x_{5}=30 \Omega$, and $x_{6}=30 \Omega$. The beam pattern is shown in Figure 9. The signals DOA information is clearly resolved from the MUSIC spectrum as shown in Figure 10.

\subsubsection{Case 4. Random load reactances}

Finally, we randomly set the reactance values to $x_{1}=0 \Omega$, $x_{2}=10 \Omega, x_{3}=0 \Omega, x_{4}=-30 \Omega, x_{5}=70 \Omega$, and $x_{6}=30 \Omega$. The MUSIC spectrum is plotted in Figure 10. From the figure we can see that if the reactance is not set ordered, they only slightly influence the MUSIC spectrum but not the performance. All the spectra reach the peak values at the respective signal directions. The beam pattern for this case is shown in Figure 11. Note that the proposed technique is used for DOA estimation and not for beam forming. So the beam pattern does not necessarily correspond to the signals directions.

From the above investigations, we conclude that the manner of load settings greatly influences the algorithm's DOAs estimation performances. When setting the reactive loads, we should avoid setting them ordered, which produces 
TABLE 1: Setting of six parasitic loads of each subset at the sampling period $\# m(m=1, \ldots, 6)$. Once the setting is decided, the values do not change during the process of the DOA estimation.

\begin{tabular}{|c|c|c|c|c|c|c|c|c|}
\hline \multirow{2}{*}{ Case } & \multicolumn{6}{|c|}{ Load reactances $(\Omega)$} & \multirow{2}{*}{ MUSIC spectrum } & \multirow{2}{*}{ DOA information } \\
\hline & $x_{1}$ & $x_{2}$ & $x_{3}$ & $x_{4}$ & $x_{5}$ & $x_{6}$ & & \\
\hline 1 & 30 & 30 & 30 & 30 & 30 & 30 & Figure 6 & Not shown in the spectrum \\
\hline 2 & 0 & 30 & 0 & 30 & 0 & 30 & Figure 8 & Not shown in the spectrum \\
\hline 3 & 0 & 30 & 0 & 30 & 30 & 30 & Figure 10 & Shown in the spectrum \\
\hline 4 & 0 & 10 & 0 & -30 & 70 & 30 & Figure 10 & Shown in the spectrum \\
\hline
\end{tabular}

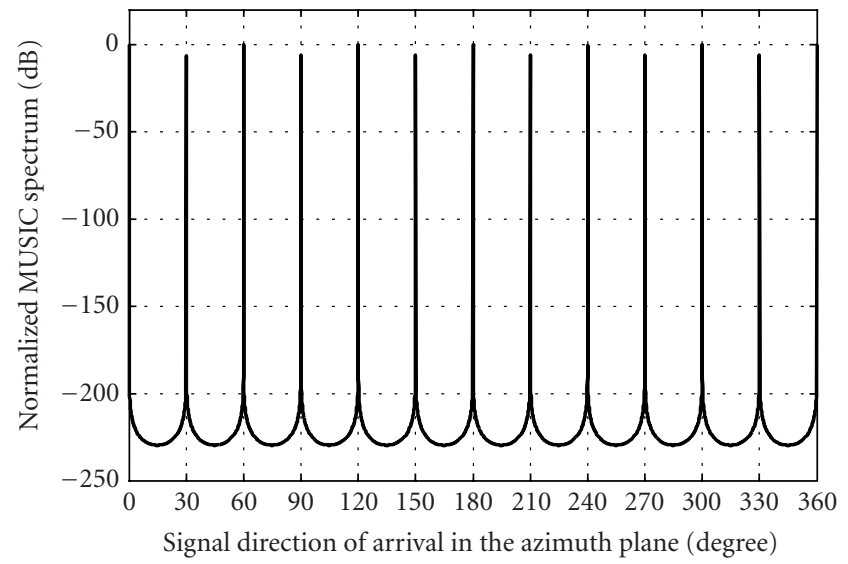

FIgURe 6: MUSIC spectrum for Case 1 is $x_{l}=30 \Omega(l=1, \ldots, 6)$, $\mathrm{SNR}=40 \mathrm{~dB}$, and the signal directions are $120^{\circ}, 150^{\circ}$, and $300^{\circ}$ in the azimuth plane.

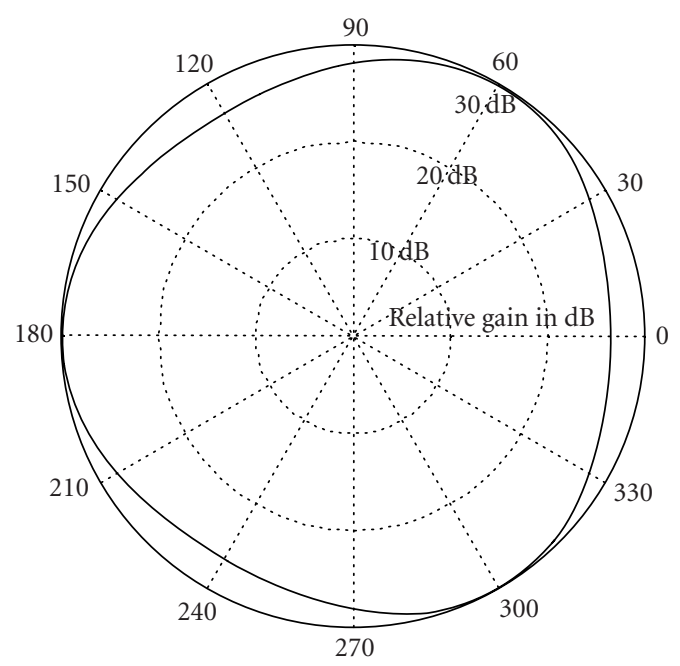

FIgURE 7: Beam pattern in the azimuth plane for the antenna sampling periods \#1 to \#6. Load setting is $x_{1}=0 \Omega, x_{2}=30 \Omega, x_{3}=0 \Omega$, $x_{4}=30 \Omega, x_{5}=0 \Omega$, and $x_{6}=30 \Omega$.

symmetric azimuthal beam patterns. With a symmetric pattern, signals impinging from different directions may produce the same output at the RF port. Analogous to linear array antenna, image peaks appear. Rather, we can set the reactance values arbitrarily to precisely estimate the DOA information.

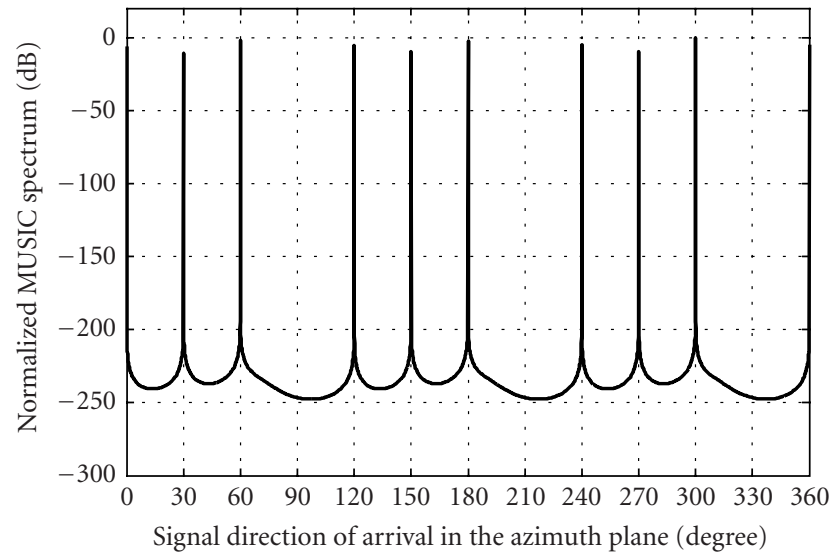

Figure 8: MUSIC spectrum for Case 2 is $x_{1}=0 \Omega, x_{2}=30 \Omega, x_{3}=$ $0 \Omega, x_{4}=30 \Omega, x_{5}=0 \Omega$, and $x_{6}=30 \Omega, \mathrm{SNR}=40 \mathrm{~dB}$, and the signal directions are $120^{\circ}, 150^{\circ}$, and $300^{\circ}$ in the azimuth plane.

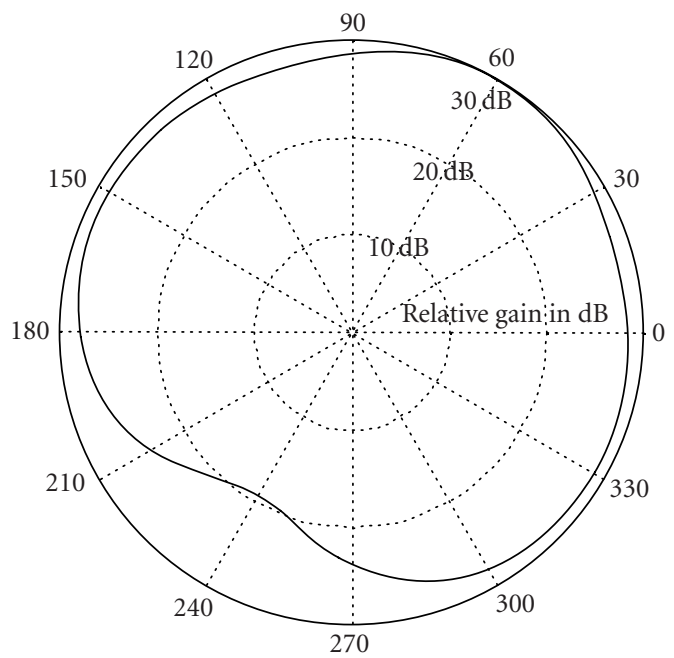

Figure 9: Beam pattern in the azimuth plane for antenna sampling periods \#1 to \#6. The load setting is $x_{1}=0 \Omega, x_{2}=30 \Omega, x_{3}=0 \Omega$, $x_{4}=30 \Omega, x_{5}=30 \Omega$, and $x_{6}=30 \Omega$.

Forming directive beam patterns is not important to the performance. The technique is dependent of the angular shifting of the beam pattern. If only the pattern is not symmetric, the DOA can be estimated correctly. However, the load setting that gives deep nulls in radiation patterns in certain directions should be avoided for the DOA estimation, 


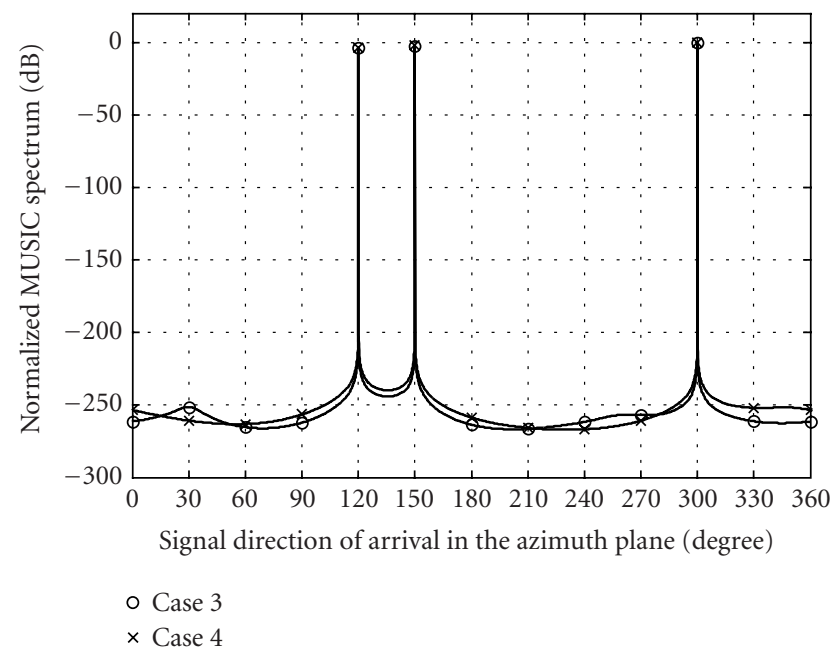

FIgUre 10: MUSIC spectra for Cases 3 and 4 . SNR $=40 \mathrm{~dB}$. The load settings are listed in Table 1 . Signal directions are $120^{\circ}, 150^{\circ}$, and $300^{\circ}$ in the azimuth plane.

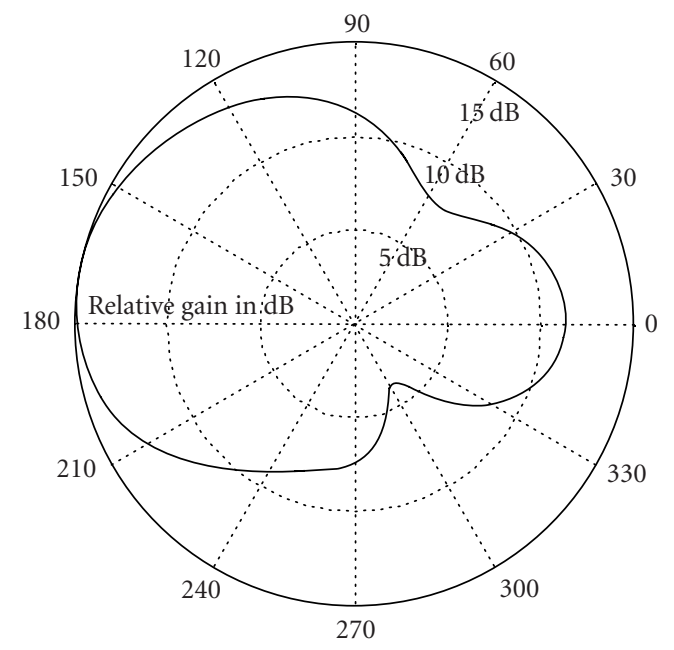

FIGURE 11: Beam pattern in the azimuth plane for antenna sampling periods \#1 to \#6. The load reactance is $x_{1}=0 \Omega, x_{2}=10 \Omega, x_{3}=0 \Omega$, $x_{4}=-30 \Omega, x_{5}=70 \Omega$, and $x_{6}=30 \Omega$.

because signals coming from that regions could be received with low power levels, or even be nulled out. Consequently, the DOA information of signal in this null region could not be extracted.

\subsection{Performance in different SNRs environments}

We also simulated the MUSIC spectra in signal environments with different SNRs. We suppose that all signal sources are totally uncorrelated and have the same power. The individual SNR for each signal source is set equal. The MUSIC spectra with 3 different SNRs from $0 \mathrm{~dB}$ to $40 \mathrm{~dB}$ are shown in Figure 12. The performance of our proposed MUSIC algorithm degrades as SNR decreases, but all three MUSIC spectra reach nearly the same peak values at the signal directions.

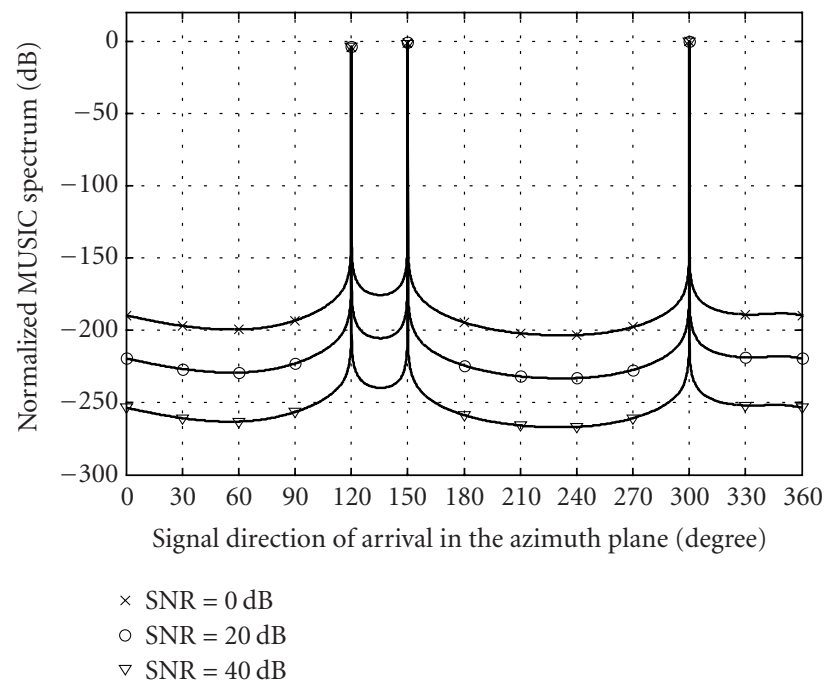

FIGURE 12: MUSIC spectra in different SNRs signal environments. Signal directions are $120^{\circ}, 150^{\circ}$, and $300^{\circ}$ in the azimuth plane. The load setting is same as in Case 4.

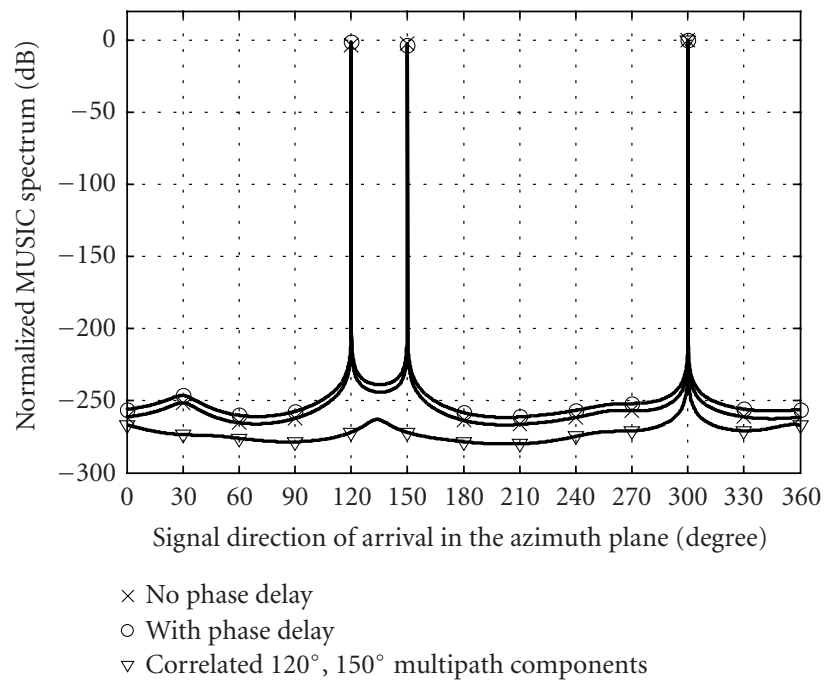

Figure 13: MUSIC spectra. The load setting is the same as for Case 4. $\mathrm{SNR}=30 \mathrm{~dB}$. Signal directions are $120^{\circ}, 150^{\circ}$, and $300^{\circ}$ in the azimuth plane.

\subsection{Performance in a multipath propagation environment}

Our study assumes a line of sight (LOS) propagation environment (Ricean channel). In a macroscopic area, where PL services, such as intelligent transportation services, are employed, base station antennas are mounted well above the roof of the buildings to provide LOS propagations between base stations and mobile terminals [27].

Ideally, signals arrive at the antenna without phase delays. The plotted MUSIC spectrum is shown in Figure 13 with a "X"-marked solid line. In a practical wireless communication environment, multipath propagation delays of the 


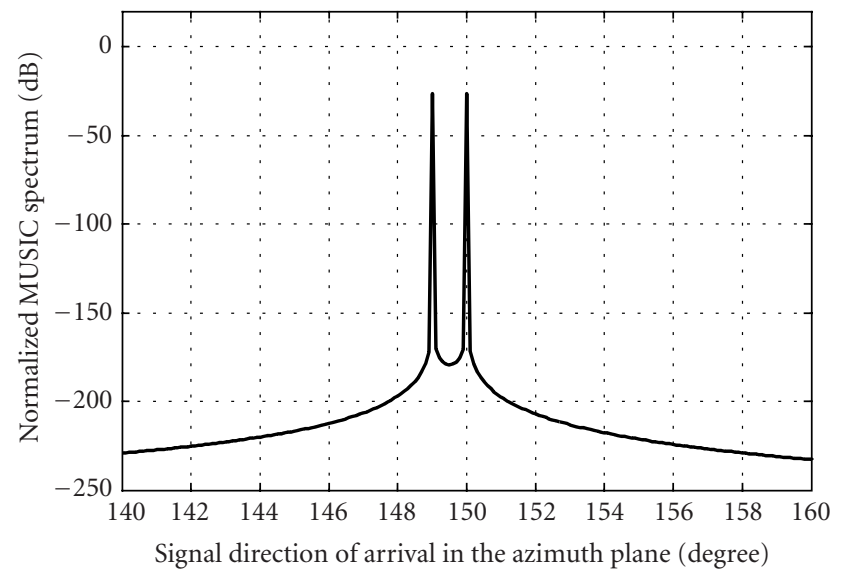

FIgURe 14: MUSIC spectrum. The load setting is the same as for Case 3. SNR $=30 \mathrm{~dB}$. Two signal directions are $149^{\circ}$ and $150^{\circ}$ in the azimuth plane, respectively.

signals are expected. Received signals contain the LOS component and the multipath components from each far field signal source. We assume that the LOS components are uncorrelated with the multipath components [28] and that the LOS components have much higher signal power level, we could still reasonably represent the received signals at the antennas as a summation of only the LOS components from different directions with phase differences induced by the propagation delays. Signal directions are set as $120^{\circ}, 150^{\circ}$, and $300^{\circ}$ in the azimuth plane. The MUSIC spectrum for delayed signals is shown in Figure 13 with a circle-marked solid line. The signal direction information is obtained from the spectrum.

In a microscopic area, the base station antennas are mounted below the rooftop level to confine the signal coverage into a small local area [29]. In this case, the base stations are located in a rich scattering environment. There are no LOS components. Signals received at the base station antenna are a collection of multipath components with angular spreads, which are possible to be correlated. Figure 13 shows the resulting MUSIC spectrum with a " $\nabla$ "-marked solid line. Two multipath components from 1 far field signal source arriving at the antenna from $120^{\circ}$ and $150^{\circ}$ in the azimuth plane are correlated. The MUSIC algorithm could not resolve correlated signals. To circumvent the rich multipath problem, the mobile terminal's spatial signature [30] could be used to locate the mobile terminal's location.

\subsection{Resolution and limitation in number of resolvable signals}

The proposed technique is capable to estimate the DOAs of two signal sources with $1^{\circ}$ angular separation. As seen from Figure 14, 2 signals impinging from $149^{\circ}$ and $150^{\circ}$ in the azimuth plane could be clearly resolved from the spectrum. The SNR is $30 \mathrm{~dB}$.

The maximum number of uncorrelated waves to be estimated is $\mathrm{M}-1 . \mathrm{M}$ is the number of subsets, the number of sampling periods, and is also the dimension of the formed signal correlation matrix $\mathbf{R}_{U U}$. The scenario is similar to the

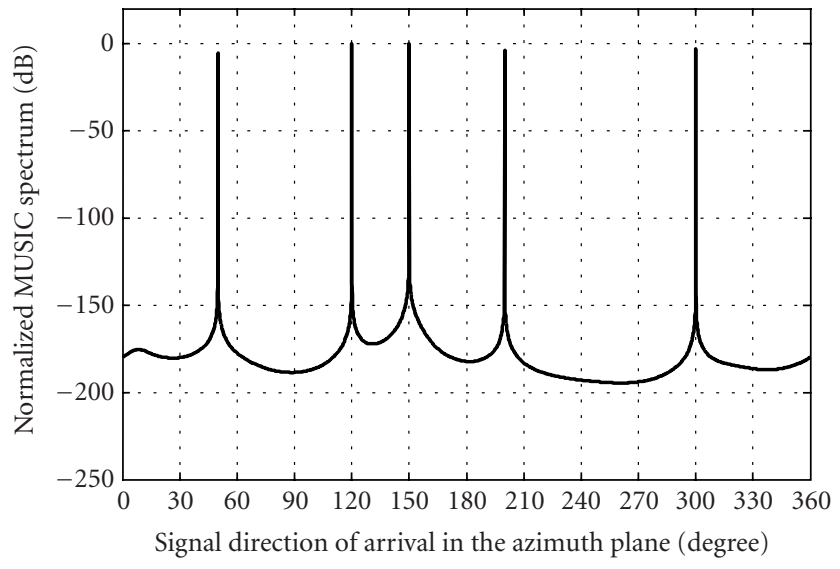

FIGURE 15: MUSIC spectrum. Load setting is the same as for Case 3. $\mathrm{SNR}=30 \mathrm{~dB}$. Signal directions are $50^{\circ}, 120^{\circ}, 150^{\circ}, 200^{\circ}$, and $300^{\circ}$ in the azimuth plane.

\begin{tabular}{|c|c|c|c|c|c|c|c|}
\hline $\begin{array}{c}\text { Periodic } \\
\text { signal } S_{1}(t)\end{array}$ & \multicolumn{3}{|c|}{$T$ signal period } & & & & \\
\hline \begin{tabular}{l|l} 
& 6 \\
\end{tabular} & 1 & 2 & 3 & 4 & 5 & 6 & 1 \\
\hline 6 & 1 & 2 & 3 & 4 & 5 & 6 & 1 \\
\hline $\begin{array}{c}\text { Periodic } \\
\text { signal } S_{2}(t)\end{array}$ & & & & & & & \\
\hline
\end{tabular}

Signal sample vector $U=\left[u_{1}(t), u_{2}(t), u_{3}(t), u_{4}(t), u_{5}(t), u_{6}(t)\right]^{T}$ periods

Figure 16: Periodic signal time slots and antenna virtual sampling periods in the proposed DOA estimation technique. Each signal contains 6 slots. The antenna rotates 6 sampling periods. Time offsets are expected in a multipath environment.

conventional MUSIC algorithm, in which $\mathbf{R}_{U U}$ with a dimension of $M$ could produce up to $M-1$ signals [23, 31]. In our design, there are 6 parasitic monopoles, thus we can estimate up to 5 DOAs as shown in Figure 15. Signal directions are set to be $50^{\circ}, 120^{\circ}, 150^{\circ}, 200^{\circ}$, and $300^{\circ}$ in azimuth plane.

\subsection{Sampling period and periodicity of transmitted signals}

Finally, we discuss the requirement of the periodicity of the transmitted signals for employing the proposed DOA estimation technique. In the derivation of $\mathbf{R}_{U U}$, we require that each individual signal source transmits periodic signals. In a practical wireless communication environment, time offsets among periodic signals and the antenna sampling periods, induced by different propagation path delays are expected.

However, the signal period does not need to be aligned with the sampling period (Figure 16). This is true because the signal source transmits signals longer than the length of total 6 sampling periods; and each signal period is the same as the antenna sampling period $T$. The received signals at the antenna are still periodic, therefore, (17) is still valid. The requirement means that when doing DOA estimation, mobile terminals will transmit periodic signals instead of message signals. With the a priori knowledge of the location of 
wireless base stations, where the antennas are mounted, and with the database of the map of the local area, PL service could be provided at the expense of a short message period. This is applicable in cases when mobile subscribers are in dangerous situations and need to make a rescue calls, such as the E999 call in Singapore and the E911 call in America. For applications in ad hoc networks where the DOA information is required for data package routing, the periodical signal could be assigned during the network setup period.

\subsection{Speed of DOA estimation}

In our design, 16 signal bits are stored at each sampling period. A minimum length of $16 \times 6=96$ bits of the periodic signals for 6 antenna sampling periods is required. For timedivision multiple-access (TDMA) IS-136 System, 1 frame comprises 6 time slots with the length of 6.67 millisecond each. In each time slot, 260 data bits are transmitted. This indicates that the DOA estimation is possible to be carried out within 1 TDMA time slot. The tuning of the reactance could be done in nanoseconds [22], and the off-the-shelf diodes can realize state switching in a few nanoseconds.

\section{CONCLUSION}

In this paper, a novel approach for executing the highresolution MUSIC algorithm based on a single RF port smart antenna has been proposed. After presenting the configuration and the working principle of the antenna, the performance of the proposed technique for various aspects have been studied. The results justified that the technique is capable for a high-resolution DOA estimation of 1 degree, which is comparable with a conventional MUSIC algorithm based on DBF array antennas. The proposed technique for DOA estimation based on a signal RF port parasitic antenna has many advantages over that on a DBF. They are as follows.

(1) In operation, only six parasitic elements are switched on. The switches consume a few milliwatt of power and the leakage current of a reverse biased diode is negligible. The SARLPA antenna features low power consumption and low complexity. It is suitable for commercial implementations at mobile terminals such as laptops in ad hoc wireless networks $[7,19]$ to provide direction information. It can also be easily mounted on vehicles to realize position-based services.

(2) Contrary to the DOA estimation based on a DBF, the mutual coupling is utilized to steer the beam [13]. Thus the modified MUSIC algorithm is free from the negative influences of the mutual coupling among antenna elements. In a DBF, this influence has to be compensated for the enhancement of system performance in a high-resolution DOA estimation as studied in $[32,33]$.

(3) The technique gives high-quality performance in low SNR signal environments. The performance in an SNR $=0 \mathrm{~dB}$ environment is shown in Figure 12.

(4) The proposed technique is very flexible in operation. The parasitic elements are reactively loaded and con- trolled with switches. The antenna can be configured into a reactively controlled directive array with a certain amount of reactance-loaded parasitic elements. Adaptive beamforming algorithm could be employed at the baseband to tune the beam direction [16] as an adaptive directional antenna helping to reduce the channel interferences and improve the channel capacity. If the directions of the impinging waves are known, the antenna could be reconfigured into a switched parasitic antenna. Beam patterns could be controlled by simply switching on and off different parasitic elements to function as a switched beam antenna, or sector antenna [2].

(5) Parasitic elements are arranged to form a circular array antenna. When used for DOA estimation, it could give the signal direction information covering $360^{\circ}$ in the azimuth plane.

Simulation studies have also pointed out that further improvements are needed to enhance system performances to make it more applicable to practical wireless communication systems. The areas of improvements are as follows.

(1) The proposed technique works in an uncorrelated signal environment. New methods should explore signal sources spatial signatures in a correlated signal environment for PL services.

(2) The proposed antenna will not suffer from the negative influence from the mutual coupling between antenna elements. However, the calibration of the antenna aperture over DOA, frequency and temperature, weather environment, and fabrication error are still unavoidable and they influence the antenna performances.

(3) The Doppler effect of the fast moving mobile terminals needs to be investigated.

(4) The antenna having 36 parasitic monopoles equispaced on the circle surrounding the active central element has been used to theoretically demonstrate the idea of eigenstructure-based direction finding algorithm with a single RF port smart antenna. For practical implementations, using less parasitic antenna elements could reduce the complexity. Furthermore, the proposed SARLPA with elements in the form of microstrip patch also renders a much compacter design.

The smart antennas, a critical technology for the third and beyond generation wireless communications, are under extensive studies. Many testbeds and field trials have been built to justify their potential benefits. Employed at the base stations, they could bring a higher-system capacity, coverage extension, and new services such as the cellular infrastructure-based PL services. At the wireless mobile terminals, they could bring the benefits as lower power consumption, reduced interference level. This paper offers an economical approach to implement smart antennas into the existing terrestrial wireless mobile communications system to serve as an essential and fundamental technique. 


\section{REFERENCES}

[1] A. Reinhardt, "The wireless challenge," Business Week, pp. 48-58, October 2003.

[2] T. S. Rappaport, J. H. Reed, and B. D. Woerner, "Position location using wireless communications on highways of the future," IEEE Communications Magazine, vol. 34, no. 10, pp. 33-41, 1996.

[3] G. V. Tsoulos, "Smart antennas for mobile communication systems: benefits and challenges," IEE Electronic \& Communication Engineering Journal, vol. 11, no. 2, pp. 84-94, 1999.

[4] K. J. Krizman, T. E. Biedka, and T. S. Rappaport, "Wireless position location: fundamentals, implementation strategies, and sources of error," in Proc. IEEE Vehicular Technology Conference, vol. 2, pp. 919-923, Phoenix, Ariz, USA, May 1997.

[5] W. C. Y. Lee, Mobile Communications Engineering, McGrawHill, New York, NY, USA, 1997.

[6] R. Ramanathan and J. Redi, "A brief overview of ad hoc networks: challenges and directions," IEEE Communications Magazine, vol. 40, no. 5, pp. 20-22, 2002.

[7] T. Ohira, "Adaptive array antenna beamforming architectures as viewed by a microwave circuit designer," in Proc. AsiaPacific Microwave Conference, pp. 828-833, Sydney, Australia, December 2000.

[8] D. V. Thiel and S. Smith, Switched Parasitic Antennas for Cellular Communications, Artech House, Norwood, Mass, USA, 2001.

[9] S. L. Preston, D. V. Thiel, T. A. Smith, S. G. O’Keefe, and J. W. $\mathrm{Lu}$, "Base-station tracking in mobile communications using a switched parasitic antenna array," IEEE Trans. Antennas and Propagation, vol. 46, no. 6, pp. 841-844, 1998.

[10] R. Vaughan, "Switched parasitic elements for antenna diversity," IEEE Trans. Antennas and Propagation, vol. 47, no. 2, pp. 399-405, 1999.

[11] D. V. Thiel, S. G. O'Keefe, and J. W. Lu, "Electronic beam steering in wire and patch antenna systems using switched parasitic elements," in Proc. IEEE Antennas and Propagation Society International Symposium, vol. 1, pp. 534-537, Baltimore, Md, USA, July 1996.

[12] S. L. Preston, D. V. Thiel, J. W. Lu, S. G. O'Keefe, and T. S. Bird, "Electronic beam steering using switched parasitic patch elements," Electronics Letters, vol. 33, no. 1, pp. 7-8, 1997.

[13] R. F. Harrington, "Reactively controlled directive arrays," IEEE Trans. Antennas and Propagation, vol. AP-26, no. 3, pp. 390-395, 1978.

[14] T. Ohira and K. Gyoda, "Hand-held microwave directionof-arrival finder based on varactor-tuned analog aerial beamforming," in Proc. Asia-Pacific Microwave Conference, vol. 2, pp. 585-588, Taipei, Taiwan, December 2001.

[15] K. Yang and T. Ohira, "ESPAR antennas-based signal processing for DS-CDMA signal waveforms in ad hoc network systems," in Proc. IEEE 3rd Workshop on Signal Processing Advances in Wireless Communications, pp. 130-133, Taoyuan, Taiwan, March 2001.

[16] J. Cheng, Y. Kamiya, and T. Ohira, "Adaptive beamforming of ESPAR antenna based on steepest gradient algorithm," IEICE Transactions on Communications, vol. E84-B, no. 7, pp. 17901800, 2001.

[17] C. Sun and N. C. Karmakar, "Adaptive beamforming of ESPAR antenna based on simultaneous perturbation stochastic approximation theory," in Proc. Asia-Pacific Microwave Conference, vol. 1, pp. 192-195, Kyoto, Japan, November 2002.

[18] C. Sun, A. Hirata, T. Ohira, and N. C. Karmakar, "Fast beamforming of electronically steerable parasitic array radiator antennas: theory and experiment," IEEE Trans. Antennas and Propagation, vol. 52, no. 7, pp. 1819-1832, 2004.
[19] N. C. Karmakar and M. E. Bialkowski, "A beam-forming network for a circular switched-beam phased array antenna," IEEE Microwave and Wireless Components Letters, vol. 11, no. 1, pp. 7-9, 2001.

[20] K. Iigusa and T. Ohira, "Reconfigurable array antenna with reactively controlled electronically invisible parasitic radiators," IEICE Tech. Rep. AP2002-122, The Institute of Electronics, Information and Communication Engineers, 2003, pp. 1924.

[21] S. A. Leonov and A. I. Leonov, Handbook of Computer Simulation in Radio Engineering, Communications, and Radar, Artech House, Norwood, Mass, USA, 2001.

[22] S. Y. Liao, Microwave Solid-State Devices, Prentice-Hall, Englewood Cliffs, NJ, USA, 1985.

[23] R. O. Schmidt, "Multiple emitter location and signal parameter estimation," IEEE Trans. Antennas and Propagation, vol. AP-34, no. 3, pp. 276-280, 1986.

[24] L. C. Godara, "Application of antenna arrays to mobile communications. II. Beam-forming and direction-of-arrival considerations," Proc. IEEE, vol. 85, no. 8, pp. 1195-1245, 1997.

[25] D. H. Johnson and D. E. Dudgeon, Array Signal Processing: Concepts and Techniques, Prentice-Hall PTR, Englewood Cliffs, NJ, USA, 1993.

[26] D. C. Cox, R. R. Murray, H. W. Arnold, A. Norris, and M. Wazowicz, "Cross-polarization coupling measured for $800 \mathrm{MHz}$ radio transmission in and around houses and large buildings," IEEE Trans. Antennas and Propagation, vol. AP-34, no. 1, pp. 83-87, 1986.

[27] W. C. Jakes, Microwave Mobile Communications, IEEE Press, Piscataway, NJ, USA, 1994.

[28] C. Tepedelenlioglu, Modeling and Mitigation of Time- and Frequency-Selective Fading in Single- and Multi-Carrier Communications, Ph.D. thesis, Department of Electrical and Computer Engineering, University of Minnesota, Minneapolis, Minn, USA, March 2001.

[29] J. Parsons, The Mobile Radio Propagation Channel, Halsted Press, New York, NY, USA, 1992.

[30] S.-S. Jeng, G. Xu, H.-P. Lin, and W. J. Vogel, "Experimental studies of spatial signature variation at $900 \mathrm{MHz}$ for smart antenna systems," IEEE Trans. Antennas and Propagation, vol. 46, no. 7, pp. 953-962, 1998.

[31] R. O. Schmidt and R. E. Franks, "Multiple source DF signal processing: An experimental system," IEEE Trans. Antennas and Propagation, vol. AP-34, no. 3, pp. 281-290, 1986.

[32] Y. Inoue, K. Mori, and H. Arai, "DOA error estimation using 2.6 GHz DBF array antenna," in Proc. Asia-Pacific Microwave Conference, vol. 2, pp. 701-704, Taipei, Taiwan, December 2001.

[33] K. R. Dandekar, H. Ling, and G. Xu, "Effect of mutual coupling on direction finding in smart antenna applications," Electronics Letters, vol. 36, no. 22, pp. 1889-1891, 2000.

Chen Sun is now pursuing the Ph.D. degree at Nanyang Technological University, Singapore. His research interests include adaptive beamforming, array signal processing, MIMO communications, and array antenna design. From November 2002 to March 2003, he was with ATR Adaptive Communications Research Laboratories, Kyoto, Japan as a student intern, working on personal wireless links for wireless ad hoc networks.

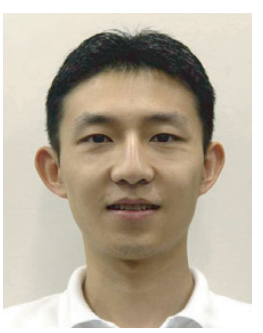

$\mathrm{He}$ was an Invited Session (Active and Adaptive Array Antennas) Cochair in Asia-Pacific Microwave Conference 2002, Kyoto, Japan. 
Nemai C. Karmakar obtained his M.S. and Ph.D. in electrical engineering from the University of Saskatchewan, Canada, and University of Queensland, Australia in 1991 and 1999, respectively. In August 1990, he was as a Research Assistant at the Communications Research Group, the University of Saskatchewan. From 1992 to 1995 he worked as a Microwave Design Engineer at

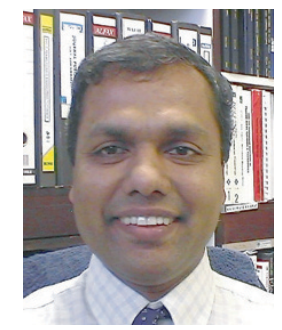
Mitec Ltd., Australia, where he contributed significantly to the development of land mobile satellite antennas for the Australian Mobilesat. He taught at Queensland University of Technology, Australia in 1995-1996. From September 1998 to March 1999 he worked as a research engineer within the Radar Laboratory, Nanyang Technological University, Singapore. Since March 1999 he is an Assistant Professor and Graduate Advisor in the Division of Communications, the School of Electrical and Electronic Engineering, Nanyang Technological University, Singapore. He has published more than 100 referred journal and conference papers and three book chapters. He is a senior member of IEEE. He served as a member of the executive committee of Singapore IEEE Section in 2000 and is now an executive committee member of IEEE APS/MTT Chapter. He is a Senior Lecturer in the Department of Electrical and Computer Systems Engineering, Monash University, Clayton Campus, Monash. 